\title{
Achados neuropatológicos da COVID-19: uma revisão sistemática
}

\author{
Neuropathological findings of COVID-19: a systematic \\ review
}

\author{
Resultados neuropatológicos del COVID-19: \\ una revisión sistemática
}

\author{
Qesya Rodrigues Ferreira ${ }^{1}$, Bruna Teixeira da Costa ${ }^{1}$, Marcelle \\ Machado Mendes ${ }^{3}$, Vinicius Nogueira Cavalcanti ${ }^{2}$, Alberto Christian \\ Luz Santos ${ }^{1}$, David Manoel Pereira Araújo ${ }^{1}$, Patrícia da Silva Oliveira ${ }^{4}$ \\ 1.Acadêmico de Medicina do Instituto Multidisciplinar em Saúde, Universidade Federal da Bahia, Vitória da \\ Conquista-BA, Brasil. \\ 2.Acadêmico de Medicina da Faculdade de Saúde Santo Agostinho, Vitória da Conquista-BA, Brasil. \\ 3.Acadêmico de Medicina da Escola Superior de Ciências da Saúde, Brasília-DF, Brasil. \\ 4.Bióloga, Doutora, docente do Instituto Multidisciplinar em Saúde, Universidade Federal da Bahia, Vitória \\ da Conquista-BA, Brasil.
}

\begin{abstract}
Resumo
Introdução. A doença causada pelo Sars-Cov-2, COVID-19, foi identificada pela primeira vez na China e apresentou rápida expansão mundial, sendo reconhecida como pandemia em março de 2020. Apesar de causar uma infecção de vias aéreas, descobertas clínicas recentes e estudos com animais sugerem que o vírus também promove vias neuronais notáveis e comprometimentos do sistema circulatório, levando a lesões no sistema nervoso central. Objetivo. Coletar e analisar os dados publicados entre 2019 e 2020 a respeito de lesões encontradas no sistema nervoso a partir de estudos de necrópsia e de imagem realizados em seres humanos. Método. Uma busca sistemática foi feita nas bases de dados PubMed e Science Direct utilizando os descritores "COVID-19" OU "SARS-CoV-2" OU "Coronavirus" AND "Central Nervous System" OU "Brain" AND "Autopsy" OR "RMI". Foram incluídos estudos originais primários, publicados entre 2019 e 2020. Foram excluídos estudos realizados em animais, casos não comprovados de contaminação por COVID-19 e estudos in vitro. Resultados. Os principais achados desta revisão sistemática apontam para causas imunológicas, inflamatórias e infecciosas. Hemorragia intracranial e intracerebral e Acidente Vascular Cerebral (AVC) isquêmico foram os principais eventos relatados, sendo que o AVC relacionado a COVID-19 tende a ser mais grave do que outras causas. Conclusão. Embora os dados científicos ainda sejam escassos para explicar as vias de dano do SARS-CoV-2 no sistema nervoso central, as evidências disponíveis sugerem uma correlação entre COVID-19 e complicações neurológicas em alguns pacientes.
\end{abstract}

Unitermos. COVID-19; Coronavírus; Neuroinflamação; Acidente Vascular Cerebral

\footnotetext{
Abstract

Introduction. COVID-19, the coronavirus disease 2019, was first identified in China and showed a rapid worldwide expansion, being recognized as a pandemic in March 2020. Its etiological agent, the severe acute respiratory syndrome coronavirus 2 (SARS-CoV-2), causes an airway infection. However, recent clinical findings and animal studies suggest that the virus also promotes notable neuronal pathways and circulatory system impairments, leading to injuries in the central nervous system. Objective. Collect and analyze data published between 2019 and 2020 regarding lesions found in the nervous system based on necropsy and image studies performed on humans. Method. A systematic search was performed in the PubMed and ScienceDirect databases using the descriptors "COVID-19" OR "SARS-CoV-2" OR "Coronavirus" AND "Central Nervous System" OR "Brain" AND "Autopsy" OR "RMI". Original primary studies, published between 2019 and 2020, were included. Animal studies, unproven cases of COVID-19 contamination, and in vitro studies were excluded. Results. Although
} 
studies of pathological mechanisms and viral dissemination methods are still scarce, the main findings of this systematic review point to immunological, inflammatory, and infectious causes. Intracranial and intracerebral hemorrhage and ischemic stroke were the main reported events, with COVID-19 stroke tend to be severer than other causes. Conclusions. Even though the scientific data is still scarce to explain SARS-CoV-2 damage pathways in the central nervous system, the evidence available suggests a correlation between COVID-19 and neurological complications in some patients.

Keywords. COVID-19; Coronavirus; Neuroinflammation; Stroke

\section{RESUMEN}

Introducción. La enfermedad causada por Sars-Cov-2, COVID-19, se identificó por primera vez en China y tuvo un costo mundial rápido, siendo reconocida como una pandemia en marzo de 2020. A pesar de causar una infección de las vías respiratorias, hallazgos clínicos recientes y estudios con animales sugieren que el virus también promueve vías neuronales notables y alteraciones del sistema circulatorio, lo que conduce a lesiones en el sistema nervioso central. Objetivo. Recopilar y analizar datos publicados entre 2019 y 2020 sobre lesiones encontradas en el sistema nervioso a partir de necropsias y estudios de imagen realizados en humanos. Método. Se realizó una búsqueda sistemática en las bases de datos PubMed y ScienceDirect utilizando los descriptores "COVID-19" O "SARS-CoV-2" O "Coronavirus" Y "Sistema Nervioso Central" O "Cerebro" Y "Autopsia" O "RMI". Se incluyeron los estudios primarios originales, publicados entre 2019 y 2020. Se excluyeron los estudios en animales, los casos no comprobados de contaminación por COVID-19 y los estudios in vitro. Resultados. Los principales hallazgos de esta revisión sistemática apuntan a causas inmunológicas, inflamatorias e infecciosas. La hemorragia intracraneal e intracerebral y el accidente cerebrovascular isquémico fueron los principales eventos notificados, y el accidente cerebrovascular relacionado con el COVID-19 tiende a ser más grave que otras causas. Conclusión. Aunque los datos científicos todavía son escasos para explicar las vías del daño del SARS-CoV-2 al sistema nervioso central, la evidencia disponible sugiere una correlación entre el COVID-19 y las complicaciones neurológicas en algunos pacientes.

Palabras clave: COVID-19; Coronavirus; Neuroinflamación; Accidente Cerebrovascular

Trabalho realizado no Instituto Multidisciplinar em Saúde, Universidade Federal da Bahia, Vitória da Conquista-BA, Brasil.

Endereço para correspondência: Qesya Rodrigues Ferreira. Instituto Multidisciplinar em Saúde, Universidade Federal da Bahia. R. Hormindo Barros 58, Candeias. CEP 45029-094. Vitória da ConquitaBA, Brasil. Email: qesya.rodrigues@ufba.br

\section{INTRODUÇÃO}

\section{O coronavírus da Síndrome Respiratória Aguda Grave 2} (SARS-CoV-2) foi identificado em dezembro de 2019 como o vírus responsável por um surto de pneumonia severa em Wuhan, na China ${ }^{1}$. Com um agente etiológico inédito e o desconhecimento das autoridades de saúde, a Doença do Coronavírus 2019 (COVID-19) se disseminou, atingindo os cinco continentes. Dessa forma, a Organização Mundial da Saúde (OMS) definiu a enfermidade como uma pandemia em 
11 de março de 2020 e atualmente, o número de infectados ultrapassa 75 milhões com mais de um milhão de óbitos².

Apesar da COVID-19 se definir fundamentalmente como uma infecção de vias aéreas, incluindo sintomas como tosse, febre, dispneia, anosmia e ageusia, o SARS-CoV-2 também possui habilidades neuro-invasivas e pode se espalhar do sistema respiratório para o sistema nervoso central ${ }^{3,4}$. As lesões encontradas no cérebro podem ocorrer por duas vias: podem ser decorrentes da infecção direta no órgão, que ocorre após a ligação do vírus com o receptor de enzima conversora de angiotensina tipo 2 (ECA-2) presente no endotélio e em células do sistema nervoso ${ }^{5}$ ou podem resultar de hipercitocinemias e de danos à barreira hematoencefálica, as quais causam, respectivamente, eventos tromboembólicos e hemorragias ${ }^{6}$. Essas lesões são potencializadas pelo aumento da pressão vascular decorrente da desregulação do sistema renina-angiotensinaaldosterona (SRAA), causada pela ligação com a ACE-2, o que corrobora para a etiologia multifatorial de tais achados ${ }^{7-}$ 9 .

Devido ao estado crítico mundial gerado pela pandemia da COVID-19 e seus possíveis acometimentos neurológicos, são necessários mais estudos que esclareçam as alterações ocorridas no Sistema Nervoso Central (SNC). Dessa forma, o objetivo deste estudo foi coletar e analisar os dados publicados entre 2019 e 2020 a respeito de lesões encontradas no sistema nervoso a partir de estudos de necrópsia e de imagem realizados em seres humanos. 


\section{MÉTODO}

\section{Estratégia de busca}

Uma busca sistemática foi realizada nas bases de dados eletrônicas PubMed e ScienceDirect no período de 04 de agosto a 17 de outubro de 2020 utilizando os seguintes descritores: "COVID-19" OR "SARS-CoV-2" OR "Coronavirus" AND "Central Nervous System" OR "Brain". Foram encontrados 382 artigos no PubMed e 413 artigos no ScienceDirect, dos quais 238 foram eliminados por duplicação. O protocolo adotado por esta revisão está sumarizado na Figura 1.

\section{Critérios para a seleção dos estudos}

Foram incluídos estudos originais primários, publicados entre 2019 e 2020, disponíveis integralmente na internet de forma gratuita, em língua inglesa, com métodos claros, cuja revisão foi executada por pares.

Foram excluídos artigos que não comprovaram a infecção por COVID-19 por meio de exames laboratoriais nos pacientes observados e que não apresentavam exames de imagem ou estudos de necropsia.

Dos 742 resultados encontrados, 243 tratava-se de estudos primários correspondentes à área de interesse escritos em inglês. Destes, 190 eram textos completos disponíveis de forma gratuita. Dos estudos restantes, 171 foram excluídos por não apresentarem exames de imagem ou estudos com necropsia ou por não citarem a execução de exame laboratorial para o diagnóstico de COVID-19. 


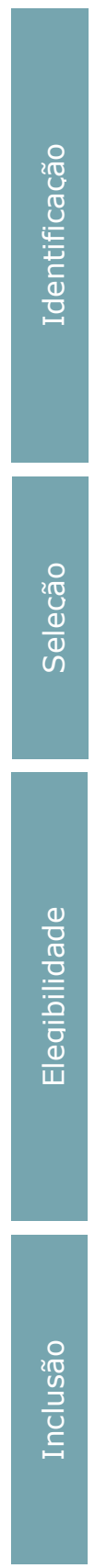

Número de relatos identificados no banco de dados de buscas

(PubMed $n=382$; ScienceDirect $n=413$ )

Número de relatos após eliminar os duplicados $(n=742)$
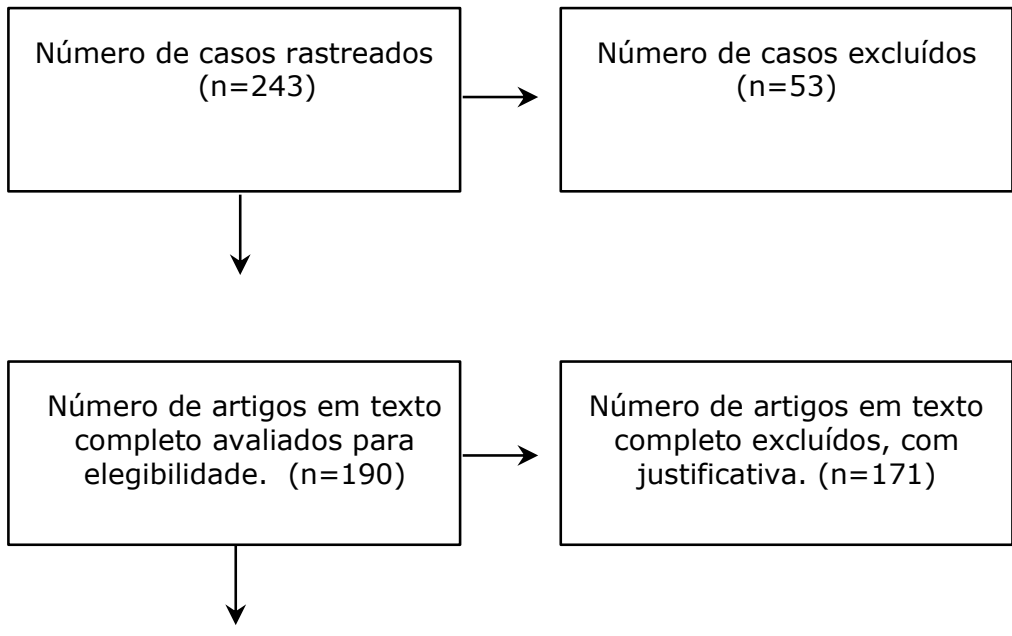

Número de artigos em texto completo excluídos, com justificativa. $(n=171)$

Número de estudos incluídos em síntese qualitativa. $(n=19)$

Número de artigos adicionados a partir das referências. $(n=11)$

Figura 1. Protocolo de seleção dos artigos elegíveis por critérios de pesquisa.

\section{1 artigos citados nas referências e considerados importantes para esta revisão foram adicionados manualmente. \\ Extração de dados}


Os dados foram sumarizados em fichamentos e posteriormente organizados em planilhas eletrônicas para realização das análises estatísticas.

\section{Análise estatística}

A análise estatística foi realizada somando-se o número de pacientes que apresentavam achados histopatológicos e de imagem semelhantes nos diferentes estudos. Por meio de gráficos gerados pelas planilhas eletrônicas, calculou-se a incidência de cada evento em 1000 pacientes.

\section{RESULTADOS}

Foram encontrados 30 artigos que atenderam aos critérios pré-estabelecidos pelo protocolo adotado nesta revisão. Os principais achados incluem alterações no bulbo olfatório, nos nervos cranianos, nas meninges, no córtex cerebral, na substância branca, no cerebelo, no corpo caloso, dentre outros. Em certos casos foram relatados achados nos grandes vasos, tálamo, hipocampo, além de diferentes giros e núcleos. Ainda foram observadas alterações medulares. As lesões constatadas incluem inflamações bulbares, tromboses, hemorragias, edemas, congestões vasculares, infartos e diversas outras manifestações graves, incluindo lesões desmielinizantes. 
As Tabelas 1 e 2 resumem, respectivamente, os resultados encontrados em estudos de necropsia e de imagem, indicando as principais estruturas afetadas. Os Gráficos 1 e 2 apresentam, respectivamente, a incidência de lesões encefálicas encontradas e a incidência por região encefálica afetada.

Tabela 1. Síntese dos resultados dos estudos histopatológicos e de necropsia em pacientes que foram infectados pelo SARS-CoV-2.

\begin{tabular}{|c|c|c|c|}
\hline Estudo & $\begin{array}{l}\text { Número de } \\
\text { pacientes }\end{array}$ & Estruturas afetadas ( $n$ ) & Achados patológicos (n) \\
\hline $\begin{array}{l}\text { Al-Dalahmah et } \\
\text { al., } 2020^{5}\end{array}$ & 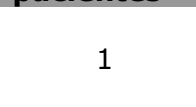 & $\begin{array}{l}\text { Leptomeninges (1); } \\
\text { Cerebelo (1); } \\
\text { Ponte (1). }\end{array}$ & $\begin{array}{l}\text { Hemorragia (1); } \\
\text { Edema }(1)\end{array}$ \\
\hline $\begin{array}{c}\text { Schurink et al., } \\
2020^{6}\end{array}$ & 9 & $\begin{array}{l}\text { Bulbo olfatório (9); } \\
\text { Medula oblonga (9); } \\
\text { Encéfalo e cerebelo (9). }\end{array}$ & $\begin{array}{l}\text { Inflamação (9); } \\
\text { AVC isquêmico }(2) ; \\
\text { Encefalite necrotizante }(1) ; \\
\text { Encefalopatia hipóxica ( } 2) ; \\
\text { Placas neutrofílicas e trombo (3); } \\
\text { Lesões por hipóxia (9); } \\
\text { Infiltrado de células T no parênquima cerebral (9). }\end{array}$ \\
\hline $\begin{array}{l}\text { Hanley et al., } \\
2020^{10}\end{array}$ & 9 & $\begin{array}{l}\text { Substância branca (5); } \\
\text { Região da artéria cerebral média } \\
\text { (1) }\end{array}$ & $\begin{array}{l}\text { Ativação microglial de moderada a intensa no SNC } \\
\text { (5); } \\
\text { Infiltrado de células T leve nos vasos e capilares } \\
\text { (5); } \\
\text { AVC hemorrágico (1) }\end{array}$ \\
\hline $\begin{array}{l}\text { Remmelink et } \\
\text { al., } 2020^{11}\end{array}$ & 11 & Meninges (5) & $\begin{array}{l}\text { Hematoma subdural (1); } \\
\text { Hemorragia cerebral }(1) ; \\
\text { Necrose focal isquêmica (3); } \\
\text { Congestão vascular e edema (5); } \\
\text { Esponginose focal e difusa }(10) ;\end{array}$ \\
\hline $\begin{array}{l}\text { Kantonen et al., } \\
2020^{12}\end{array}$ & 4 & $\begin{array}{l}\text { Região perivascular (4); } \\
\text { Substância branca (1); } \\
\text { Mesencéfalo (1); } \\
\text { Ponte (1); } \\
\text { Medula espinhal (1). }\end{array}$ & $\begin{array}{l}\text { Degradação perivascular (4); } \\
\text { Hemorragia perivascular (1); } \\
\text { Doença vascular }(1) ; \\
\text { Lesão por hipóxia (4) }\end{array}$ \\
\hline $\begin{array}{c}\text { Reichard et al., } \\
2020^{13}\end{array}$ & 1 & $\begin{array}{l}\text { Vasos sanguíneos (1); } \\
\text { Substância branca (1). }\end{array}$ & $\begin{array}{l}\text { Lesões vasculares }(1) ; \\
\text { Lesões desmielinizantes }(1) ; \\
\text { Lesões hemorrágicas intraparenquimatosas } \\
\text { Lesão axonal }(1) ; \\
\text { Encefalomielite disseminada aguda - (ADEM)-like } \\
(1) ; \\
\text { Focos de necrose microscópicas focais em } \\
\text { substância branca }(1) .\end{array}$ \\
\hline $\begin{array}{l}\text { Matschke et al. } \\
2020^{14}\end{array}$ & 43 & $\begin{array}{l}\text { Território da artéria cerebral } \\
\text { posterior, artéria cerebral anterior } \\
\text { e artéria cerebral média. (6); } \\
\text { Tronco encefálico (4); } \\
\text { Cerebelo ( } 3) ; \\
\text { Meninges ( } 3) ; \\
\text { Bulbo olfatório (8) }\end{array}$ & $\begin{array}{l}\text { AVC isquêmico (6); } \\
\text { Astrogliose ( } 37) ; \\
\text { Infiltrado de linfócitos T citotóxicos nas meninges } \\
\text { (34); } \\
\text { Proteínas virais em nervos cranianos do tronco } \\
\text { encefálico ( } 21) \text {. } \\
\text { Edema (24). }\end{array}$ \\
\hline
\end{tabular}


Tabela 1 (cont.). Síntese dos resultados dos estudos histopatológicos e de necropsia em pacientes que foram infectados pelo SARS-CoV-2.

\begin{tabular}{|c|c|c|c|}
\hline Estudo & $\begin{array}{l}\text { Número de } \\
\text { pacientes }\end{array}$ & Estruturas afetadas (n) & Achados patológicos $(\mathbf{n})$ \\
\hline $\begin{array}{l}\text { Fabbri et al., } \\
2020^{15}\end{array}$ & 10 & $\begin{array}{l}\text { Lobo frontal. (2) } \\
\text { Meninges (10) } \\
\text { Lobo parietal (1); } \\
\text { Uncos (2) }\end{array}$ & $\begin{array}{l}\text { Lesões hipóxico-isquêmicas (10) } \\
\text { AVE isquêmico (3) } \\
\text { Congestão meníngea (10); } \\
\text { Acúmulo purulento meníngeo (1) } \\
\text { Extravasamento focal de sangue na meninge } \\
\text { subaracnóide }(1) ; \\
\text { Herniação do uncos (2); } \\
\text { Edema (10) } \\
\text { Presença do RNA viral no nervo olfatório e no } \\
\text { tecido cerebral (1) } \\
\text { Trombos intravasculares intraparenquimais (10) }\end{array}$ \\
\hline $\begin{array}{c}\text { Jensen et al., } \\
2020^{16}\end{array}$ & 2 & $\begin{array}{l}\text { Vasos sanguíneos (1); } \\
\text { Tronco encefálico (1); } \\
\text { Cerebelo (2). }\end{array}$ & $\begin{array}{l}\text { Infarto cortical multifocal severo (1) } \\
\text { Calcificações perivasculares (1); } \\
\text { Encefalite no tronco encefálico, principalmente na } \\
\text { região do bulbo dorsal }(1) \\
\text { Infarto subagudo em córtex cerebelar }(1) ; \\
\text { AVC isquêmico (2) }\end{array}$ \\
\hline $\begin{array}{l}\text { Kirschenbaum } \\
\text { et al., } 2020^{17}\end{array}$ & 4 & $\begin{array}{l}\text { Hipotálamo (1) } \\
\text { Córtex frontal (2) } \\
\text { Córtex temporal (1) } \\
\text { Córtex parietal (1) } \\
\text { Gânglios da base (1) } \\
\text { Ponte (1) } \\
\text { Corpo caloso (2) }\end{array}$ & $\begin{array}{l}\text { Edema difuso (1) } \\
\text { Aterosclerose leve e irregular (1) } \\
\text { Neurônio com degeneração granulovacuolar no } \\
\text { hipocampo (1) } \\
\text { Atrofia difusa leve (1) } \\
\text { Hemorragia pontual (2) } \\
\text { Endotelite ( } 2 \text { ) } \\
\text { Calcificação no giro denteado do hipocampo (1) } \\
\text { Trombose intravascular (1) } \\
\text { Micro-trombose intravascular (1) } \\
\text { Isquemia subaguda (1) }\end{array}$ \\
\hline
\end{tabular}

Tabela 2. Síntese dos resultados dos estudos de imagem em pacientes que foram infectados pelo SARS-CoV-2.

\begin{tabular}{|c|c|c|c|}
\hline Estudo & $\begin{array}{l}\text { Número de } \\
\text { pacientes }\end{array}$ & Estruturas afetadas (n) & Achados patológicos (n) \\
\hline $\begin{array}{l}\text { Kandemirli et al., } \\
2020^{18}\end{array}$ & 27 & $\begin{array}{l}\text { Substância branca (3) } \\
\text { Lobo frontal ( } 4 \text { ) } \\
\text { Lobo parietal (3) } \\
\text { Lobo occipital (4) } \\
\text { Lobo temporal (1) } \\
\text { Córtex insular (3) } \\
\text { Giro do cíngulo (3) }\end{array}$ & $\begin{array}{l}\text { Anormalidade de intensidade de sinal cortical } \\
(10) ; \\
\text { Anormalidade de intensidade do sinal da } \\
\text { substância branca profunda e subcortical } \\
\text { (3); } \\
\text { Trombose de seios venosos aguda ( } 1) ; \\
\text { Infarto da artéria cerebral direita média (1). }\end{array}$ \\
\hline Kremer et al., $2020^{19}$ & 37 & $\begin{array}{l}\text { Lobo temporal medial (16) } \\
\text { Substância branca }(20) \text {. } \\
\text { Corpo caloso }(2) \text {. } \\
\text { Pedúnculo cerebelar }(2)\end{array}$ & $\begin{array}{l}\text { Lesões multifocais associadas a hemorragias } \\
\text { na substância branca }(11) ; \\
\text { Micro hemorragias isoladas na substância } \\
\text { branca (9); } \\
\text { Lesões hemorrágicas intracerebrais (20); } \\
\text { Anormalidade de sinal no lobo temporal } \\
\text { medial (16); } \\
\text { Lesão hiperintensa no corpo caloso (2); } \\
\text { Encefalopatia necrotizante aguda (2). }\end{array}$ \\
\hline $\begin{array}{l}\text { Moriguchi et al., } \\
2020^{20}\end{array}$ & 1 & $\begin{array}{l}\text { Corno inferior do ventrículo lateral } \\
\text { direito; } \\
\text { Lobo temporal direito; } \\
\text { Hipocampo. }\end{array}$ & $\begin{array}{l}\text { Alterações de sinal hiperintensas no lobo } \\
\text { temporal medial direito e hipocampo (1); } \\
\text { Atrofia hipocampal (1); } \\
\text { Ventriculite lateral direita (1); } \\
\text { Encefalite, principalmente no lobo medial } \\
\text { direito e no hipocampo (1). }\end{array}$ \\
\hline
\end{tabular}

Tabela 2 (cont.). Síntese dos resultados dos estudos de imagem em pacientes que foram infectados pelo SARS-CoV-2. 


\begin{tabular}{|c|c|c|c|}
\hline Estudo & $\begin{array}{l}\text { Número de } \\
\text { pacientes }\end{array}$ & Estruturas afetadas (n) & Achados patológicos (n) \\
\hline $\begin{array}{l}\text { Romero-Sánchez et } \\
\text { al., } 2020^{21}\end{array}$ & 841 & $\begin{array}{l}\text { Lobo occipital (4); } \\
\text { Lobo parietal (4); } \\
\text { Lobo temporal (1); } \\
\text { Lobo medial (1). }\end{array}$ & $\begin{array}{l}\text { Lesões hiperintensas subcorticais bilaterais } \\
\text { (1) } \\
\text { Edema vasogênico (1) } \\
\text { encefalopatia reversível posterior (1); } \\
\text { AVC isquêmico ( } 2) ; \\
\text { Hemorragia intracraniana (2); } \\
\text { Vasculite do SNC (1) }\end{array}$ \\
\hline $\begin{array}{l}\text { Radmanesh et al., } \\
2020^{22}\end{array}$ & 242 & $\begin{array}{l}\text { Substância branca. (134); } \\
\text { Circulação anterior (9); } \\
\text { Circulação posterior (4); } \\
\text { Grandes vasos (4). }\end{array}$ & $\begin{array}{l}\text { Alterações inespecíficas compatíveis com } \\
\text { microangiopatia. (134); } \\
\text { AVC isquêmico (13); } \\
\text { Hemorragia intracraniana aguda (11). }\end{array}$ \\
\hline $\begin{array}{l}\text { Radmanesh et al., } \\
2020^{23}\end{array}$ & 11 & $\begin{array}{l}\text { Giro pré-central da substância } \\
\text { branca justacortical (10); } \\
\text { Lobos occipitais. ( } 7) ; \\
\text { Substância branca justacortical } \\
\text { (5); } \\
\text { Corpo caloso. (4). }\end{array}$ & $\begin{array}{l}\text { Hiperintensidades simétricas e confluentes } \\
\text { compatíveis com leucoencefalopatia (10); } \\
\text { Micro hemorragias (7). }\end{array}$ \\
\hline $\begin{array}{l}\text { Morassi et al., } \\
2020^{24}\end{array}$ & 6 & $\begin{array}{l}\text { Áreas corticais e subcorticais de } \\
\text { ambos os hemisférios cerebrais } \\
(2) ; \\
\text { Giro cingulado (1); } \\
\text { Tálamo direito }(1) ; \\
\text { Centro semioval }(1) ; \\
\text { Núcleo caudado direito (1); } \\
\text { Giro frontal médio esquerdo (1). } \\
\text { Cerebelo (1) }\end{array}$ & $\begin{array}{l}\text { AVC isquêmico. (4); } \\
\text { AVC hemorrágico (2); } \\
\text { Hipodensidade multifocal (6); } \\
\text { Área leptomeníngea cortical de realce no } \\
\text { giro frontal médio esquerdo (1); } \\
\text { Hemorragias em ambos os hemisférios } \\
\text { cerebelares com compressão do IV } \\
\text { ventrículo e tronco encefálico (1); } \\
\text { Edema cerebral difuso (1) } \\
\text { Hemorragia frontal direita com extensão } \\
\text { para o sistema ventricular (1). }\end{array}$ \\
\hline Zoghi et al., $2020^{25}$ & 1 & $\begin{array}{l}\text { Medula (cervical e torácica) (1); } \\
\text { Pedúnculos cerebrais e ponte (1); } \\
\text { Esplênio do corpo caloso (1). }\end{array}$ & $\begin{array}{l}\text { Mielite }(1) ; \\
\text { Hiperintensidade de padrão heterogêneo } \\
\text { marmorizado no corpo caloso (1). }\end{array}$ \\
\hline $\begin{array}{l}\text { Klironomos et al., } \\
2020^{26}\end{array}$ & 47 & $\begin{array}{l}\text { Lobo frontal (1). } \\
\text { Cerebelo (1). } \\
\text { Artéria carótida interna distal (6). } \\
\text { Corpo caloso ( } 34) \text {. } \\
\text { Região subaracnoide (9). } \\
\text { Substância branca (23) }\end{array}$ & $\begin{array}{l}\text { Hematomas intraparenquimatosos. (2); } \\
\text { Lesão citotóxica ( } \\
1) ; \\
\text { Hemorragia (11); } \\
\text { Anormalidades de sinal (59); } \\
\text { AVC isquêmico (10); }\end{array}$ \\
\hline $\begin{array}{l}\text { Anzalone et al., } \\
2020^{27}\end{array}$ & 4 & $\begin{array}{l}\text { Envolvimento multifocal do córtex } \\
\text { (4) }\end{array}$ & $\begin{array}{l}\text { Encefalopatia subaguda com "padrão" entre } \\
\text { todos eles }(4) \\
\text { Hiperintensidade cortical (4) }\end{array}$ \\
\hline Efe et al., $2020^{28}$ & 1 & Lobo temporal esquerdo (1) & $\begin{array}{l}\text { Sinal hiperintenso no lobo temporal } \\
\text { esquerdo (1). }\end{array}$ \\
\hline Sirous et al., $2020^{29}$ & 1 & $\begin{array}{l}\text { Tonsila cerebelar descendente (1); } \\
\text { Tronco cerebelar (1); } \\
\text { IV ventrículo (1). }\end{array}$ & $\begin{array}{l}\text { Apagamento de sulco cerebral difuso (1); } \\
\text { Edema cerebral (1); } \\
\text { Herniação tonsilar cerebelar descendente, } \\
\text { com compressão do tronco encefálico e } \\
\text { estreitamento do IV ventrículo (1); } \\
\text { Constrição arterial cerebral difusa e seio } \\
\text { venoso dural (1) } \\
\text { Encefalopatia (1) }\end{array}$ \\
\hline AlKetbi et al., $2020^{30}$ & 1 & $\begin{array}{l}\text { Substância cinzenta das regiões } \\
\text { cervical, dorsal e lombar da } \\
\text { medula espinhal (1). }\end{array}$ & $\begin{array}{l}\text { Sinal hiperintenso (1) } \\
\text { Mielite aguda resultante de danos do quadro } \\
\text { pós-infeccioso. (1) }\end{array}$ \\
\hline TunÇ et al., $2020^{31}$ & 4 & $\begin{array}{l}\text { Região à esquerda da artéria } \\
\text { cerebral média. (1); } \\
\text { Região lenticuloestriada da artéria } \\
\text { cerebral esquerda (1); } \\
\text { Ponte (1); } \\
\text { Região da artéria cerebral medial } \\
\text { (1). }\end{array}$ & AVC isquêmico (4). \\
\hline Afshar et al., $2020^{32}$ & 1 & $\begin{array}{l}\text { Tálamo bilateral. (1); } \\
\text { Lobo temporal medial. (1); } \\
\text { Ponte (1). }\end{array}$ & $\begin{array}{l}\text { Encefalite parainfecciosa }(1) ; \\
\text { Altas intensidades de sinal em tálamo } \\
\text { bilateral, temporal medial e ponte (1). }\end{array}$ \\
\hline
\end{tabular}

Tabela 2 (cont.). Síntese dos resultados dos estudos de imagem em pacientes que foram infectados pelo SARS-CoV-2. 


\begin{tabular}{|c|c|c|c|}
\hline Estudo & $\begin{array}{l}\text { Número de } \\
\text { pacientes }\end{array}$ & Estruturas afetadas (n) & Achados patológicos (n) \\
\hline $\begin{array}{l}\text { Poyiadji et al., } \\
2020^{33}\end{array}$ & 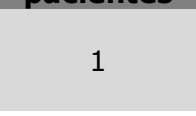 & $\begin{array}{l}\text { Tálamo bilateral }(1) ; \\
\text { Lobos temporais mediais (1); } \\
\text { Regiões subinsulares }(1) .\end{array}$ & $\begin{array}{l}\text { Encefalopatia hemorrágica aguda } \\
\text { necrotizante (1) }\end{array}$ \\
\hline Politi et al., $2020^{34}$ & 1 & $\begin{array}{l}\text { Giro reto direito }(1) \\
\text { Bulbos olfatórios }(1)\end{array}$ & $\begin{array}{l}\text { Hiperintensidade cortical no giro reto direito } \\
(1) ; \\
\text { Hiperintensidade sutil no bulbo olfatório (1). }\end{array}$ \\
\hline $\begin{array}{l}\text { Virhammar et al., } \\
2020^{35}\end{array}$ & 1 & $\begin{array}{l}\text { Região subinsular (1); } \\
\text { Tálamo }(1) ; \\
\text { Tronco cerebral }(1) ; \\
\text { Lobos temporais mediais }(1) ; \\
\text { Hipocampo }(1) ; \\
\text { Pendúnculos cerebrais }(1) ; \\
\text { Ponte }(1) \text {. }\end{array}$ & $\begin{array}{l}\text { Áreas de baixa atenuação no tálamo e } \\
\text { mesencéfalo }(1) ; \\
\text { Sinais de ruptura da barreira } \\
\text { hematoencefálica (1). } \\
\text { Sinal patológico simétrico compatível com } \\
\text { Encefalite Necrosante Aguda (1); } \\
\text { Aumentos de contraste no tálamo central e } \\
\text { região subinsular (1). }\end{array}$ \\
\hline $\begin{array}{l}\text { Guennec et al., } \\
2020^{36}\end{array}$ & 1 & $\begin{array}{l}\text { Córtex pré-frontal orbital direito } \\
\text { adjacente ao bulbo olfatório (1); } \\
\text { Córtex pré-frontal medial direito } \\
\text { (1); } \\
\text { Núcleo caudado direito (1) }\end{array}$ & $\begin{array}{l}\text { Hiperintensidade de sinal do córtex pré- } \\
\text { frontal orbital direito adjacente ao bulbo } \\
\text { olfatório (1). }\end{array}$ \\
\hline Palao et al., $2020^{37}$ & 1 & $\begin{array}{l}\text { Lobo temporal esquerdo (1); } \\
\text { Lobo occiptal direito }(1)\end{array}$ & $\begin{array}{l}\text { Lesões desmielinizantes periventriculares } \\
\text { supratentoriais (1). }\end{array}$ \\
\hline
\end{tabular}

Gráfico 1. Incidência de lesões encefálicas em 1000 pacientes de acordo com o estudo histopatológico e de necrópsia.

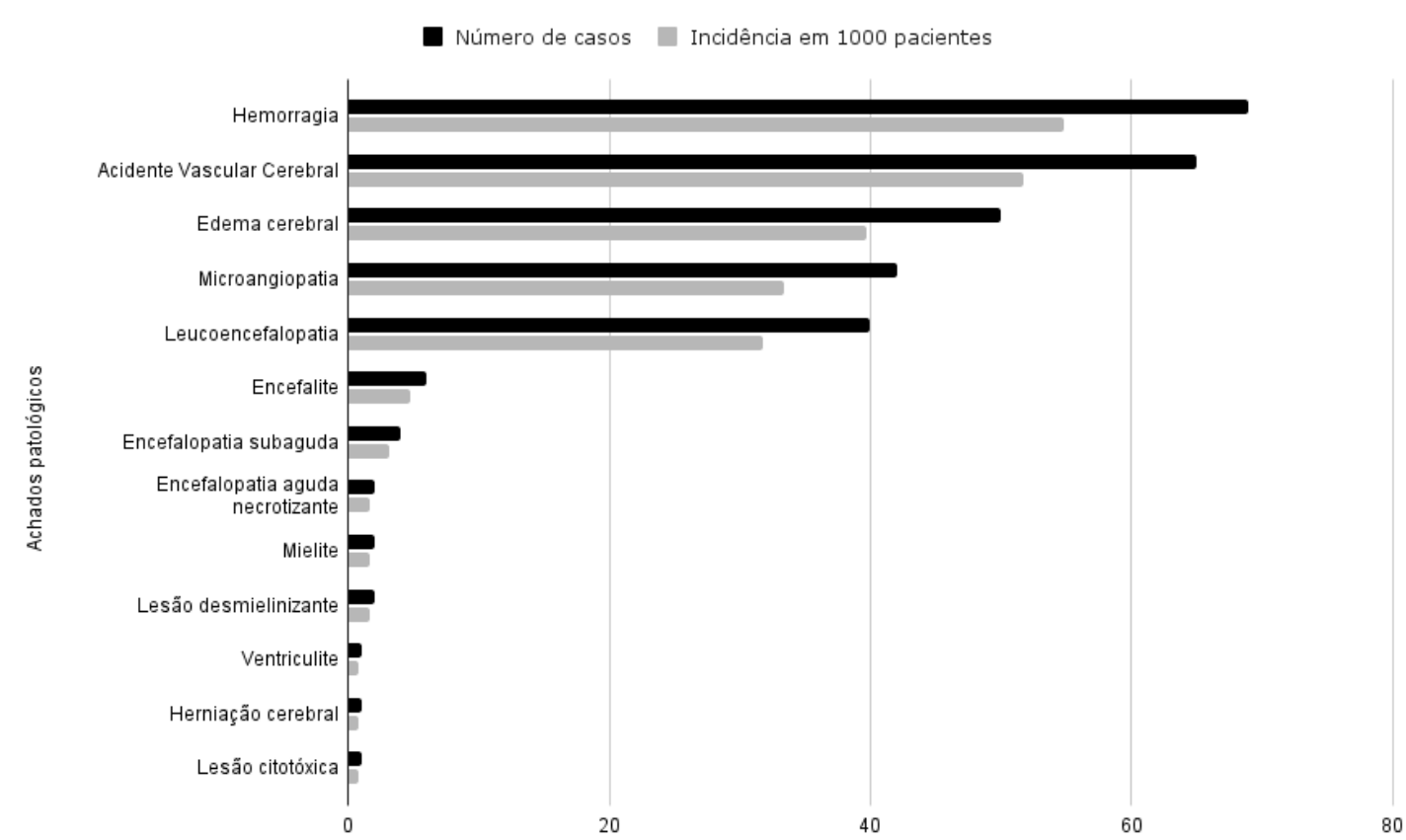


Gráfico 2. Incidência de lesões, por área encefálica afetada, em 1000 pacientes de acordo com os estudos de imagem.

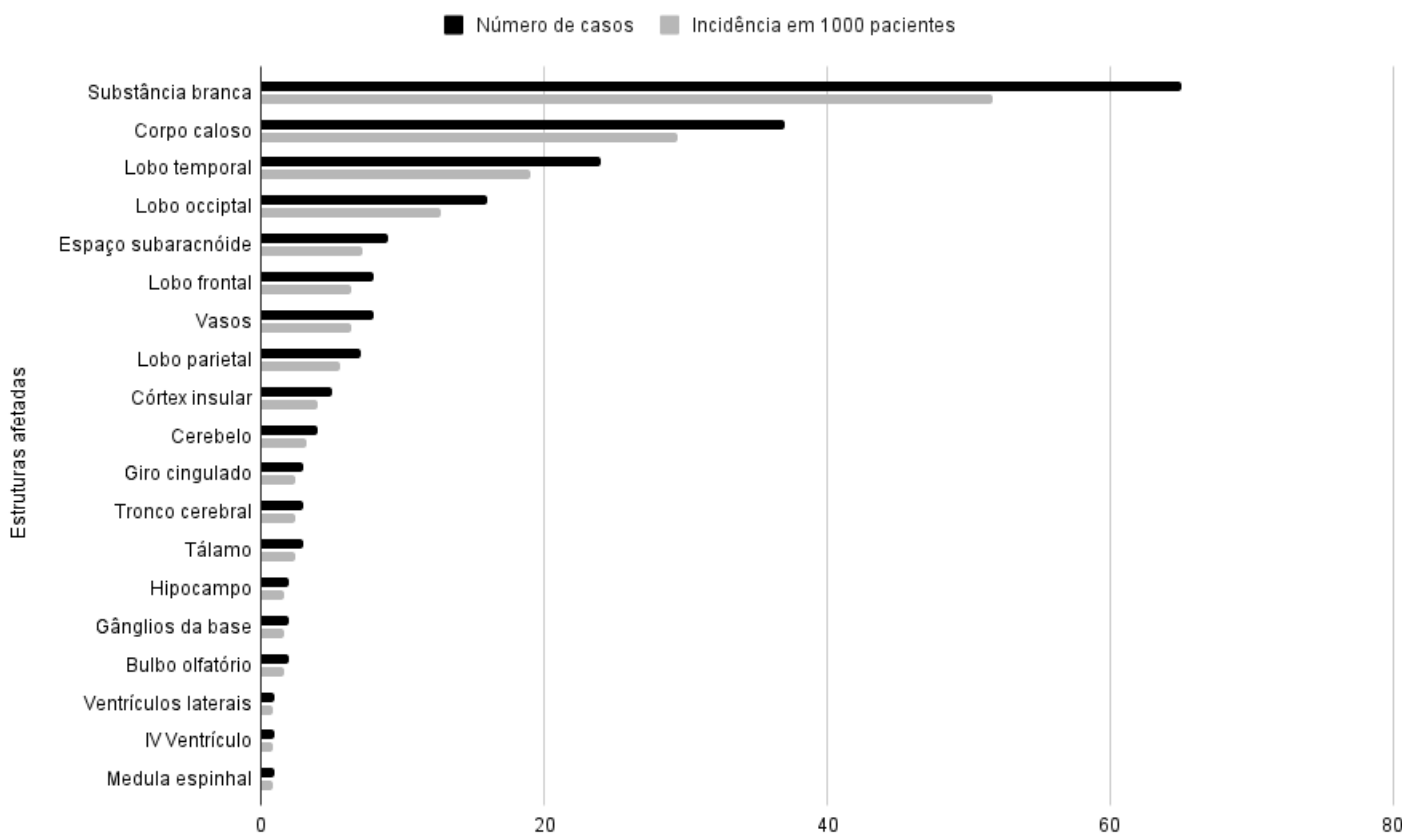

\section{DISCUSSÃO}

O SARS-CoV-2 contamina células epiteliais e nervosas a partir da conexão entre as glicoproteínas spike, em seu envelope, com a enzima conversora de angiotensina (ECA$2)^{38,39}$. Além de estar amplamente disponível na barreira hematoencefálica, facilitando a entrada do vírus no sistema nervoso, essa enzima tem grande ação vasoconstritora, o que favorece a formação de trombos ${ }^{31,40}$.

Hemorragias intracranianas e intracerebrais foram extensamente relatadas, destacando-se a presença de micro-hemorragias $5,11,19,21-23,26,41$. Há indicações de que a COVID-19 grave pode estar relacionada ao risco de hemorragias microvasculares multifocais e lesões isquêmicas na substância branca subcortical e profunda. Essas lesões podem levar ao coma, déficits neurológicos focais ${ }^{42}$ e 
acidentes vasculares cerebrais ${ }^{43,44}$. A hemorragia pode interferir com a função cerebral por meio de mecanismos, incluindo a destruição ou a compressão do tecido cerebral dado o efeito de massa que ocorre no interior do crânio ${ }^{32}$. A hemorragia intraventricular, ainda, pode levar à dilatação ventricular, o que contribui para esse efeito ${ }^{32}$.

Os achados presentes nessa revisão confirmam a associação fisiopatológica entre COVID-19 e acidente vascular cerebral (AVC) isquêmico5,11,19-23,26,41, por sua vez associados pelos autores a um estado pró-trombótico promovido pela infecção viral, visto que os exames laboratoriais de pacientes em um estágio avançado da doença constantemente apresentam altos níveis de Dímero$D$, um produto da degradação da fibrina ${ }^{45}$. AVCs isquêmicos associados à COVID-19 estão associados com piores resultados e altas taxas de mortalidade quando comparados a outras causas ${ }^{46}$. Lesões endoteliais causadas pela entrada direta do SARS-CoV-2 no endotélio por meio do receptor ACE2 e a tempestade de citocinas promovida por esse processo são responsáveis por eventos cerebrovasculares, conforme apontado por um estudo sueco ${ }^{35}$. Esses dados são corroborados pela presença do vírus no líquido cefalorraquidiano (LCR) ${ }^{35}$.

Além dos achados encefálicos, também foram relatados casos de alteração medular ${ }^{12}$ e mielite 25,30 . A COVID-19 também aumenta o risco de encefalopatias como síndromes inflamatórias do SNC, hemorragia, necrose, distúrbios neurológicos periféricos, encefalomielite disseminada aguda 
$(A D E M)$, síndrome de Guillain-Barré (SGB), entre outros. Essas são condições que podem predispor a doenças cerebrovasculares, como AVCs isquêmicos ${ }^{7-9}$. Ainda que se trate de sequelas neurológicas relativamente pouco prevalentes na população analisada, os dados mais recentes apontam para a imprescindibilidade de acompanhamento e vigilância de infectados.

Descargas neuronais foram encontradas em pacientes infectados, provocando sintomas como convulsões e/ou crises epilépticas $27,28,32$, os quais são mais frequentes no curso clínico da doença ${ }^{47,48}$. As lesões ao SNC provocadas pelos efeitos virais podem causar sintomas nos nervos periféricos (por exemplo, oftálmico e maxilar) que geram sintomas como distúrbios no paladar (ageusia e hipogeusia) e olfato (anosmia, hiposmia e disosmia) ${ }^{49}$.

A encefalite foi observada por muitos pesquisadores ${ }^{10,22,34,50}$. Essa condição se caracteriza por espessamento perivascular, infiltração linfocítica e proliferação microglial, envolvendo principalmente as regiões subcorticais de substância cinzenta. Acredita-se que isto seja decorrente de uma resposta imune exacerbada do SNC. Em estudo realizado na França, os casos de encefalites destrincharam-se em encefalite límbica, necrotização hemorrágica aguda e casos de encefalite diversa, além de outros achados individuais ${ }^{51}$.

Também foram identificados aumentos nos níveis de proteínas e leucócitos no $\mathrm{LCR}^{6,10}$, confirmando a presença de citocinas e quimiocinas nesta região. A nível celular foi 
identificada grande presença de partículas virais nos astrócitos e células dopaminérgicas ${ }^{40}$. Apesar de os neurônios corticais e as células microgliais apresentarem baixos níveis de infecção, a micróglia contribui para a patogênese cerebral da COVID-19 a partir da tempestade de citocinas e quimiocinas no SNC ${ }^{52}$. Essa tempestade causa lesões em estruturas medulares e na barreira hematoencefálica. A barreira prejudicada torna-se permissiva a passagem viral, que invade primeiramente as estruturas da base do cérebro. Devido à natureza inflamatória dessa invasão, nota-se que a participação das células microgliais e dos astrócitos na história natural da doença não é mediada pela contaminação direta dessas células, mas pelo aumento do estado trombofílico dos pacientes enfermos ${ }^{39}$.

Embora os resultados mostrem frequentes lesões no SNC dentro do espectro clínico de COVID-19, ainda não é possível afirmar seu verdadeiro mecanismo. A maior parte dos autores relatou que SARS-CoV-2 não estava presente no líquido cefalorraquidiano $29,36,37$, e apenas um deles confirmou a presença do vírus no $\mathrm{SNC}^{20}$.

Devido à maioria dos estudos contidos nesta revisão se basearem em relatos de caso (21 estudos, ou 70\%), e não em estudos randomizados controlados, seus resultados não podem ser entendidos como universais. A desvinculação entre a ocorrência de lesões cerebrais e as características determinantes particulares dos pacientes analisados, como comorbidades, fatores de risco e idade, pode comprometer a 
interpretação dos achados. A reduzida literatura disponível acerca dos mecanismos de neurotropismo do SARS-CoV-2 também é algo que dificulta a análise. No entanto, é possível garantir que a infecção por SARS-CoV-2 pode levar a distúrbios transicionais nas funções cerebrais causados por descargas neuronais anormais, bem como distúrbios do estado mental em pacientes com predisposições conhecidas.

\section{CONCLUSÃO}

Embora os estudos a respeito das lesões ao sistema nervoso causadas pela COVID-19 ainda sejam escassos, as evidências disponíveis sugerem uma correlação entre o COVID-19 e os danos neurológicos em alguns pacientes.

A compreensão de que doenças neurológicas podem ocorrer em um momento posterior ao quadro clínico infeccioso é uma forma de controlar comorbidades futuras e de planejar o melhor tratamento. Essa nova informação destaca as severas repercussões do coronavírus no organismo com efeito de médio a longo prazo.

Compreende-se que o número de casos aumenta a cada dia, trazendo sintomatologias e achados histológicos inéditos, sendo importante destacar a funcionalidade dos estudos de coorte e análises de casos com mais de 10 pacientes que testaram positivo para a contaminação pelo vírus, visto que estes analisam uma diversidade de condições fisiológicas, gerando resultados mais confiáveis. Também são necessários mais estudos patológicos realizados em 
humanos, dada a quantidade de dados encontrados in vitro e em animais.

\section{REFERÊNCIAS}

1.Ludwig S, Zarbock A. Coronaviruses and SARS-CoV-2: A Brief Overview. Anesth Analg 2020;131:93-6. http://dx.doi.org/10.1213/ANE.0000000000004845

2.Coronavirus disease (COVID-19). World Health Organization (endereço na Internet). Acessado em 12/2020). Disponível em: https://www.who.int/emergencies/diseases/novel-coronavirus2019? gclid=CjwKCAjwrKr8BRB EiwA7eFapjv8OZ u6sHDmH9GDT30e 5uo1lyInyKfUpJ0q5oQagPAVuYYojb4PxoC8RAQAvD BwE

3.Jin $\mathrm{Y}$, Yang $\mathrm{H}$, Ji W, Wu W, Chen S, Zhang W, et al. Virology, Epidemiology, Pathogenesis, and Control of COVID-19. Viruses 2020;12:372. http://dx.doi.org/10.3390/v12040372

4. Mao L, Jin $\mathrm{H}$, Wang $M, \mathrm{Hu} \mathrm{Y}$, Chen $\mathrm{S}$, He $\mathrm{Q}$, et al. Neurologic Manifestations of Hospitalized Patients With Coronavirus Disease 2019 in Wuhan, China. JAMA Neurol 2020;77:683-90. http://dx.doi.org/10.1001/jamaneurol.2020.1127

5.Al-Dalahmah O, Thakur KT, Nordvig AS, Prust ML, Roth W, Lignelli A, et al. Neuronophagia and microglial nodules in a SARS-CoV-2 patient with cerebellar hemorrhage. Acta Neuropathol Commun 2020;8:1-7. https://doi.org/10.1186/s40478-020-01024-2

6.Schurink B, Roos E, Radonic T, Barbe E, Bouman CSC, de Boer $\mathrm{HH}$, et al. Viral presence and immunopathology in patients with lethal COVID-19: a prospective autopsy cohort study. Lancet Microbe 2020;1:e290-9. http://dx.doi.org/10.1016/S2666-5247(20)30144-0

7.Paterson RW, Brown RL, Benjamin L, Nortley R, Wiethoff S, Bharucha $\mathrm{T}$, et al. The emerging spectrum of COVID-19 neurology: clinical, radiological and laboratory findings. Brain 2020;143:3104-20. http://dx.doi.org/10.1093/brain/awaa240

8.Dalakas MC. Guillain-Barré syndrome: The first documented COVID19-triggered autoimmune neurologic disease. Neurol Neuroimmunol Neuroinflamm $2020 ; 7: e 781$. http://dx.doi.org/10.1212/nxi.0000000000000781

9.Bellon M, Schweblin C, Lambeng N, Cherpillod P, Vazquez J, Lalive $\mathrm{PH}$, et al. Cerebrospinal Fluid Features in Severe Acute Respiratory Syndrome Coronavirus 2 (SARS-CoV-2) Reverse Transcription Polymerase Chain Reaction (RT-PCR) Positive Patients. Clin Infect Dis 2020: ciaa1165. http://dx.doi.org/10.1093/cid/ciaa1165

10. Hanley B, Naresh KN, Roufosse C, Nicholson AG, Weir J, Cooke GS, et al. Histopathological findings and viral tropism in UK patients with severe fatal COVID-19: a post-mortem study. Lancet Microbe 2020;1:e245-53. http://dx.doi.org/10.1016/S2666-5247(20)30115-4 11.Remmelink M, De Mendonça R, D'Haene N, De Clercq S, Verocq C, Lebrun $L$, et al. Unspecific post-mortem findings despite multiorgan viral spread in COVID-19 patients. Crit Care 2020;24:495. 
http://dx.doi.org/10.1186/s13054-020-03218-5

12.Kantonen J, Mahzabin S, Mäyränpää MI, Tynninen O, Paetau A, Andersson N, et al. Neuropathologic features of four autopsied COVID19 patients. Brain Pathol 2020;30:1012-6. http://dx.doi.org/10.1111/bpa.12889

13. Reichard RR, Kashani KB, Boire NA, Constantopoulos E, Guo Y, Lucchinetti CF. Neuropathology of COVID-19: a spectrum of vascular and acute disseminated encephalomyelitis (ADEM)-like pathology. Acta Neuropathol 2020;140:1-6. http://dx.doi.org/10.1007/s00401-02002166-2

14. Matschke J, Lütgehetmann M, Hagel C, Sperhake JP, Schröder AS, Edler C, et al. Neuropathology of patients with COVID-19 in Germany: a post-mortem case series. Lancet Neurol 2020;19:919-29. http://dx.doi.org/10.1016/S1474-4422(20)30308-2

15.Fabbri VP, Foschini MP, Lazzarotto T, Gabrielli L, Cenacchi G, Gallo $C$, et al. Brain ischemic injury in COVID-19-infected patients: a series of 10 post-mortem cases. Brain Pathol 2020;e12901. http://dx.doi.org/10.1111/bpa.12901

16.Jensen MP, Le Quesne J, Officer-Jones L, Teodòsio A, Thaventhiran J, Ficken $C$, et al. Neuropathological findings in two patients with fatal COVID-19. Neuropathol Appl Neurobiol 2020;47:17-25. http://dx.doi.org/10.1111/nan.12662

17. Kirschenbaum D, Imbach LL, Rushing EJ, Frauenknecht KBM, Gascho D, Ineichen BV, et al. Intracerebral endotheliitis and microbleeds are neuropathological features of COVID-19. Neuropathol Appl Neurobiol 2021;47:454-9. http://dx.doi.org/10.1111/nan.12677 18. Kandemirli SG, Dogan L, Sarikaya ZT, Kara S, Akinci C, Kaya D, et al. Brain MRI Findings in Patients in the Intensive Care Unit with COVID-19 Infection. Radiology 2020;201697. http://dx.doi.org/10.1148/radiol.2020201697

19.Kremer S, Lersy F, de Sèze J, Ferré J-C, Maamar A, Carsin-Nicol B, et al. Brain MRI Findings in Severe COVID-19: A Retrospective Observational Study. Radiology 2020;202222. http://dx.doi.org/10.1148/radiol.2020202222

20.Moriguchi T, Harii N, Goto J, Harada D, Sugawara H, Takamino J, et al. A first case of meningitis/encephalitis associated with SARSCoronavirus-2. Int J Infect Dis 2020;94:55-8. http://dx.doi.org/10.1016/j.ijid.2020.03.062

21.Romero-Sánchez CM, Díaz-Maroto I, Fernández-Díaz E, SánchezLarsen Á, Layos-Romero A, García-García J, et al. Neurologic manifestations in hospitalized patients with COVID-19: The ALBACOVID registry. Neurology 2020;95:e1060-70. http://dx.doi.org/10.1212/WNL.0000000000009937

22.Radmanesh A, Raz E, Zan E, Derman A, Kaminetzky M. Brain Imaging Use and Findings in COVID-19: A Single Academic Center Experience in the Epicenter of Disease in the United States. Am J Neuroradiol 2020;41:1179-83. http://dx.doi.org/10.3174/ajnr.A6610 23. Radmanesh A, Derman A, Lui YW, Raz E, Loh JP, Hagiwara M, et al. COVID-19-associated Diffuse Leukoencephalopathy and 
Microhemorrhages.

http://dx.doi.org/10.1148/radiol.2020202040

24.Morassi M, Bagatto D, Cobelli M, D'Agostini S, Gigli GL, Bnà C, et al. Stroke in patients with SARS-CoV-2 infection: case series. J Neurol 2020;267:2185-92. http://dx.doi.org/10.1007/s00415-020-09885-2

25.Zoghi A, Ramezani M, Roozbeh M, Darazam IA, Sahraian MA. A case of possible atypical demyelinating event of the central nervous system following COVID-19. Mult Scler Relat Disord 2020;44:102324. http://dx.doi.org/10.1016/j.msard.2020.102324

26. Klironomos S, Tzortzakakis A, Kits A, Öhberg C, Kollia E, Ahoromazdae $A$, et al. Nervous System Involvement in Coronavirus Disease 2019: Results from a Retrospective Consecutive Neuroimaging Cohort. Radiology 2020;297:E324-34. http://dx.doi.org/10.1148/radiol.2020202791

27.Anzalone N, Castellano A, Scotti R, Scandroglio AM, Filippi M, Ciceri $\mathrm{F}$, et al. Multifocal laminar cortical brain lesions: a consistent MRI finding in neuro-COVID-19 patients. J Neurol 2020;267:2806-9. http://dx.doi.org/10.1007/s00415-020-09966-2

28.Efe IE, Aydin OU, Alabulut A, Celik O, Aydin K. COVID19-Associated Encephalitis Mimicking Glial Tumor. World Neurosurger 2020;140:46-8. http://dx.doi.org/10.1016/j.wneu.2020.05.194

29.Sirous R, Taghvaei R, Hellinger JC, Krauthamer AV, Mirfendereski S. COVID-19-associated encephalopathy with fulminant cerebral vasoconstriction: CT and MRI findings. Radiol Case Rep 2020;15:220812. http://dx.doi.org/10.1016/j.radcr.2020.08.024

30. AlKetbi R, AINuaimi D, AlMulla M, AlTalai N, Samir M, Kumar N, et al. Acute myelitis as a neurological complication of Covid-19: A case report and MRI findings. Radiol Case Rep 2020;15:1591-5. http://dx.doi.org/10.1016/j.radcr.2020.06.001

31.TunÇ A, ÜnlÜbaŞ Y, Alemdar M, AkyÜz E. Coexistence of COVID-19 and acute ischemic stroke report of four cases. J Clin Neurosci 2020;77:227-9. http://dx.doi.org/10.1016/j.jocn.2020.05.018

32. Afshar H, Yassin Z, Kalantari S, Aloosh O, Lotfi T, Moghaddasi M, et al. Evolution and resolution of brain involvement associated with SARSCoV2 infection: A close Clinical - Paraclinical follow up study of a case. Mult Scler Relat Disord 2020;43:102216. http://dx.doi.org/10.1016/j.msard.2020.102216

33.Poyiadji N, Shahin G, Noujaim D, Stone M, Patel S, Griffith B. COVID-19-associated Acute Hemorrhagic Necrotizing Encephalopathy: CT and MRI Features. Radiology 2020;296:E119-20. http://dx.doi.org/10.1148/radiol.2020201187

34.Politi LS, Salsano E, Grimaldi M. Magnetic Resonance Imaging Alteration of the Brain in a Patient With Coronavirus Disease 2019 (COVID-19) and Anosmia. JAMA Neurol 2020;77:1028. http://dx.doi.org/10.1001/jamaneurol.2020.2125

35.Virhammar J, Kumlien E, Fällmar D, Frithiof R, Jackmann S, Sköld $\mathrm{MK}$, et al. Acute necrotizing encephalopathy with SARS-CoV-2 RNA confirmed in cerebrospinal fluid. Neurology 2020;95:445-9. http://dx.doi.org/10.1212/WNL.0000000000010250 
36.Guennec LL, Le Guennec L, Devianne J, Jalin L, Cao A, Galanaud D, et al. Orbitofrontal involvement in a neuroCOVID-19 patient. Epilepsia 2020;61:e90-4. http://dx.doi.org/10.1111/epi.16612

37.Palao M, Fernández-Díaz E, Gracia-Gil J, Romero-Sánchez CM, DíazMaroto I, Segura T. Multiple sclerosis following SARS-CoV-2 infection. Mult Scler Relat Disord 2020;45:102377.

http://dx.doi.org/10.1016/j.msard.2020.102377

38. Hoffmann $M$, Kleine-Weber $H$, Schroeder $S$, Krüger $N$, Herrler $T$, Erichsen S, et al. SARS-CoV-2 Cell Entry Depends on ACE2 and TMPRSS 2 and Is Blocked by a Clinically Proven Protease Inhibitor. Cell 2020;181:271-80. http://dx.doi.org/10.1016/j.cell.2020.02.052

39.Al-Olama M, Rashid A, Garozzo D. COVID-19-associated meningoencephalitis complicated with intracranial hemorrhage: a case report. Acta Neurochir 2020;162:1495-9. http://dx.doi.org/10.1007/s00701-020-04402-w

40. Yang L, Han Y, Nilsson-Payant BE, Gupta V, Wang P, Duan X, et al. A Human Pluripotent Stem Cell-based Platform to Study SARS-CoV-2 Tropism and Model Virus Infection in Human Cells and Organoids. Cell Stem Cell 2020;27:125-36.e7. http://dx.doi.org/10.1016/j.stem.2020.06.015

41.Kirschenbaum D, Imbach LL, Rushing EJ, Frauenknecht KBM, Gascho D, Ineichen BV, et al. Intracerebral endotheliitis and microbleeds are neuropathological features of COVID-19. Neuropathol Appl Neurobiol 2020;47:454-9. http://dx.doi.org/10.1111/nan.12677 42. Conklin J, Frosch MP, Mukerji S, Rapalino O, Maher M, Schaefer PW, et al. Cerebral Microvascular Injury in Severe COVID-19. medRxiv 2020.07.21.20159376.

http://dx.doi.org/10.1101/2020.07.21.20159376

43. Roy $D$, Hollingworth $M$, Kumaria $A$. A case of malignant cerebral infarction associated with COVID-19 infection. Br J Neurosurg 2020;14. http://dx.doi.org/10.1080/02688697.2020.1779180

44. He J, Cheng G, Xu W, Zhang L, Zeng Z. [Diagnosis and treatment of an elderly patient with secondary cerebral infarction caused by COVID-19]. Nan Fang Yi Ke Da Xue Xue Bao 2020;40:351-2. http://dx.doi.org/10.12122/j.issn.1673-4254.2020.03.10

45.Zhang L, Yan X, Fan Q, Liu H, Liu X, Liu Z, et al. D-dimer levels on admission to predict in-hospital mortality in patients with Covid-19. J Thromb Haemost

http://dx.doi.org/10.1111/jth.14859

46. Ntaios G, Michel P, Georgiopoulos G, Guo Y, Li W, Xiong J, et al. Characteristics and Outcomes in Patients With COVID-19 and Acute Ischemic Stroke: The Global COVID-19 Stroke Registry. Stroke 2020;51:e254-8. http://dx.doi.org/10.1161/STROKEAHA.120.031208 47.Pinna P, Grewal P, Hall JP, Tavarez T, Dafer RM, Garg R, et al. Neurological manifestations and COVID-19: Experiences from a tertiary care center at the Frontline. J Neurol Sci 2020;415:116969. http://dx.doi.org/10.1016/j.jns.2020.116969

48. Lu L, Xiong W, Liu D, Liu J, Yang D, Li N, et al. New onset acute symptomatic seizure and risk factors in coronavirus disease 2019: A 
retrospective multicenter study. Epilepsia 2020;61:e49-53. http://dx.doi.org/10.1111/epi.16524

49. Vaira LA, Deiana G, Fois AG, Pirina P, Madeddu G, De Vito A, et al. Objective evaluation of anosmia and ageusia in COVID-19 patients: Single-center experience on 72 cases. Head Neck 2020;42:1252-8. http://dx.doi.org/10.1002/hed.26204

50.Kandemirli SG, Dogan L, Sarikaya ZT, Kara S, Akinci C, Kaya D, et al. Brain MRI Findings in Patients in the Intensive Care Unit with COVID-19 Infection. Radiology 2020;297:E232-5. http://dx.doi.org/10.1148/radiol.2020201697

51.Kremer S, Lersy F, Anheim M, Merdji H, Schenck M, Oesterlé $H$, et al. Neurologic and neuroimaging findings in patients with COVID-19: A retrospective multicenter study. Neurology 2020;95:e1868-82. http://dx.doi.org/10.1212/WNL.0000000000010112

52.Lavi E, Cong L. Type I astrocytes and microglia induce a cytokine response in an encephalitic murine coronavirus infection. Exp Mol Pathol 2020;115:104474.

http://dx.doi.org/10.1016/j.yexmp.2020.104474 